\title{
An Open Agent-Based Model to Simulate the Effect of WOM Marketing Campaigns
}

\author{
Poster \\ Paul Leger Manuela López Carmen Hidalgo-Alcázar \\ Escuela de Ciencias Empresariales, Universidad Católica del Norte, Chile
} \{pleger, mlopezp, mchidalgo\}@ucn.cl

\author{
Hiroaki Fukuda \\ Shibaura Institute of Technology, Japan \\ hiroaki@shibaura-it.ac.jp
}

\begin{abstract}
Word-Of-Mouth (WOM) is the opinion of consumers about a product. There is currently a clear trend in the use of WOM to diffuse information about a new product, known as WOM marketing campaigns. Marketing researchers are studying the impact of the different types of these campaigns has. The difficulty in getting data and isolating the effect that is analyzed limits the research of marketers in this topic. Lastly, some simulation models based on agents have overcome previous difficulty. However, these models are ad-hoc and specific solutions for every study. This poster proposes an open implementation of an agent-based model to simulate different scenarios of WOM marketing campaigns. Through customizations, this proposal will allow marketing researchers to test WOM marketing campaigns in different scenarios.
\end{abstract}

\section{Categories and Subject Descriptors}

D.3.3 [Programming Languages]: Language Constructs and Features; I.2.0 [Artificial Intelligence]: General; J.1.6 [Computer Applications] Computer Applications Marketing.

\section{General Terms}

Languages, Design.

\section{Keywords}

Word-Of-Mouth, Agent-Based Models, Open Implementations.

\section{INTRODUCTION}

Before purchasing a product or service, consumers usually ask their family or friends, or even consult other consumers' opinions. In marketing, these opinions are known as Word of Mouth (WOM), which has become the most influential and credible information source for consumers [1]. Hence, companies are interested in using WOM as a new communication tool to influence consumers' decisions [2]. Using the Internet or through an offline channel, companies carry out WOM marketing campaigns: give a message to a set of consumers (a.k.a. seeds) to spread the word about a product to other consumers [2]. The goal of these campaigns is to reach a fast and high diffusion of a message.

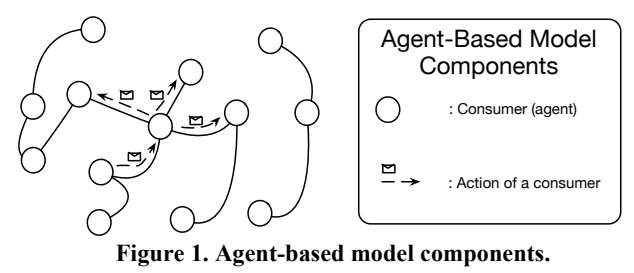

In the state-of-the-art, we can find studies that have analyzed what message characteristics and type of seeds affect consumers' intention to transmit a message in a network [2][3][4]. Other studies have examined the reasons why a message has been highly diffused [5]. Unfortunately, each study requires collecting a large amount of data from social network sites such as Facebook or many questionnaires. In addition, it is difficult to isolate the effect that we would like to analyze by using the prior techniques. To overcome these difficulties, a simulation model based on agents can be used [6], which basically simulates the diffusion for a WOM campaign. In this campaign a message is given to some consumers (agents), who make the decision whether spreading the message to their contacts or not. Some simulation models have been proposed [6][7][8], specifically tailored to fit particular characteristics of a campaign, where it is not possible to customize these characteristics. Thereby, for each new campaign, developers must $a$ ) modify in contorted ways an existing model or b) implement a new agent-based simulation model from scratch; bringing a slow development and prone-error implementations.

This paper proposes OMC, an open implementation [9] of an agent-based simulation model to measure the effect of a WOM marketing campaign. With simple configurations or code extensions, OMC will allow developers to instantiate existing models or create new ones that fit particular and unforeseen characteristics of a campaign. In OMC, developers will be able to customize strategies in messages, seeds, network, and reporting outputs of a WOM marketing campaign.

\section{BACKGROUND}

We start this section discussing each component of a WOM marketing campaign, and then we briefly explain how to use agent-based models to simulate these campaigns.

\subsection{WOM Marketing Campaign Components}

To develop a successful campaign, this company has to make decisions on the following components:

Network. A campaign must work through a channel. The channel can be online (e.g., Facebook) or offline. This channel will determine the network (with its features) in which the campaign will work.

Message. A message can consist of only information (text, URL links, images, videos) or a product with the aim of spreading the word about it. Companies can also give an incentive to encourage consumers in WOM. Recent research has examined the 


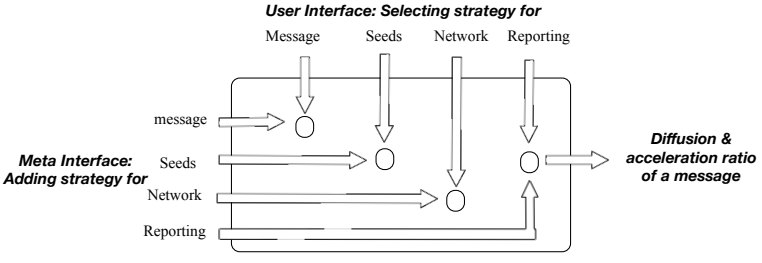

Figure 2. Potential open points of OMC.

characteristics that a message should have in order to be much diffused [5].

Seeds. Companies have a different number of seeds available. These seeds can have different characteristics: hubs (wellconnected consumers), opinion leaders (influential consumers), or bridges (connectors of two otherwise unconnected parts of a network) [3].

\subsection{AGENT-BASED MODEL}

An agent-based simulation model simulates aggregate consequences based on local interactions between individual members of a population [10]. This kind of model consists of an environment, where a set of agents are in one of a finite number of possible states, updated in discrete time steps according to local interaction rules. Figure 1 illustrates the main components of an agent-based simulation model:

Environment. The container where agents interact with others is the environment. Depending on the scenario, the environment can simulate an online network (e.g., Social Network Site, networks of email contacts) or offline network (e.g., neighbors of a city).

Agents. Apart of internal states, agents have different properties and behaviors. For example, a type of agent can have a higher influence over other agent.

Action. Using a set of local rules, agents take actions that potentially affect other agents in each discrete time step. For example, an agent can take the action of (re)sending a message.

A variety of agent-based models have been proposed to simulate WOM marketing campaigns [6][8]. Each model sets up previous components to fit particular characteristics of a campaign.

\section{PROBLEM STATEMENT}

The black box principle (expose the functionality but hide the implementation of a program) offers benefits like reuse, location of changes, and understandability. However, the application of this principle can bring issues of adaptability, performance, and reuse in unforeseen scenarios of a program. As a consequence, developers have to "code around" the program or end up creating an entirely different version of the same program [9].

Existing implementations of agent-based models to simulate WOM marketing campaigns follow (in an ad hoc manner) the black box principle. Thereby, for each campaign with different characteristics, developers must "code around" existing models in contorted ways or create a new version from scratch. As a consequence, the development of these models becomes slow development and prone-error.

\section{PROPOSAL}

This paper proposes OMC, an agent-based model to simulate WOM marketing campaigns that follows open implementation guidelines [9]. OMC will allow developers to customize crucial components used to simulate these campaigns, and at the same time, still hiding details of its implementation. As agent-based model, we concretely propose using Cellular Automata (CA) [10] because its neighborhood concept (direct contact of a consumer in OMC) allows an agent to make a decision only considering its neighbors. In addition, $\mathrm{CA}$ has been used in simulations of complex networks [11].

Figure 2 shows a big picture the core open points of OMC. In this Figure, we can see that this proposal provides a user and metainterface. The user interface allows developers to select one of available strategies for message, seeds, network, and reporting outputs. The meta-interface offers the opportunity to customize and add strategies for these four components of a WOM marketing campaign. As an example of adding a strategy, consider a new type of seed: fringe (a consumer with few connections) [3]. Using the meta-interface, developers, for example, can define the average of connections of a fridge seed and its behavior when it receives a message.

\section{PLAN}

The construction of OMC depends on two tracks. First, we will study the literature in marketing to collect other crucial points that should be open in OMC. Second, we will define abstractions for the user and meta-interface. For the user interface, we will probably construct domain-specific abstractions (or -languages) according to the marketing concepts. Instead, the meta-interface will use the full power of a Turing-complete language (e.g., objects, first-class functions). Among other languages, the Scala language brings together needs of both interfaces because of its flexibility, expressiveness, and meta-programming power to define and create new abstractions.

We plan to validate it through the emulation of some of existing agent-based models used to simulate WOM marketing campaigns.

\section{REFERENCES}

[1] J. Arndt. Role of product-related conversations in the diffusion of a new product. Journal of Marketing Research, 4(3), 291-295, 1967.

[2] R.V. Kozinets, K. De Valck, A.C. Wojnicki, and S.J.S Wilner. Networked narratives: understanding word-of-mouth marketing in online communities. Journal of Marketing, 74(2), 71-89, 2010.

[3] O. Hinz, B. Skiera, C. Barrot, and J.U. Becker. Seeding strategies for viral marketing: An empirical comparison. Journal of Marketing, 75 (6), 55-71, 2011

[4] J. E. Phelps, R. Lewis, L. Mobilio, D. Perry, and D. Raman. Viral marketing or electronic word-of-mouth advertising: Examining consumer responses and motivations to pass along email. Journal of advertising research, 44(4), 333 $348,2004$.

[5] K. Swani, B. P. Brown, and G. R. Milne. Should tweets differ for B2B and B2C? An analysis of Fortune 500 companies' Twitter communications. Industrial Marketing Management, 43(5), 873-881, 2014.

[6] S. Delre, W. Jager, T.H.A. Bijmolt, and M. A. Janssen. Will it spread or not? The effects of social influences and network topology on innovation diffusion. Journal of Product Innovation and Management, 27(2), 267-282, 2010.

[7] J. Goldenberg, B. Libai, and E. Muller. Talk of the network: A complex systems look at the underlying process of word-of-mouth. Marketing letters, 12(3), 211-223, 2001.

[8] B. Libai, E. Muller, and R. Peres. Decomposing the value of word-of-mouth seeding programs: Acceleration versus expansion. Journal of marketing research, 50(2), 161-176, 2013.

[9] G. Kiczales, J. Lamping, G. Murphy, C. V. Lopes, C. Maeda, and A. Mendhekar. Open Implementation Design Guidelines. Proceedings of the 1997 International Conference on at the Software Engineering, 481-490, 1997.

[10] J. von Neumann, Theory of Self-Reproducing Automata. (A. W. Burks, Ed.). Champaign, IL, USA: University of Illinois Press, 1966.

[11] D. Smith, J. Onnela, C. Fan, M. Fricker, and N. Johnson. Network Automata: Coupling Structure and Function in Dynamic Networks. Advances in Complex Systems, 14(3), 317-339, 2011. 